https://doi.org/10.15407/ujpe63.4.317

N.P. MALOMUZH, ${ }^{1}$ K.S. SHAKUN, ${ }^{2}$ A.A. KUZNETSOVA ${ }^{2}$

${ }^{1}$ I.I. Mechnikov National University of Odessa

(2, Dvoryanskaya Str., Odessa 65026, Ukraine; e-mail: mnp@onu.edu.ua)

2 Odessa National Maritime Academy

(8, Didrikhson Str., Odessa 65029, Ukraine; e-mail: gluon@meta.ua)

\title{
NEW POSSIBILITIES PROVIDED BY THE ANALYSIS OF THE MOLECULAR VELOCITY AUTOCORRELATION FUNCTION IN LIQUIDS
}

\begin{abstract}
Long-time tails of the molecular velocity autocorrelation function (VACF) in liquid argon at temperatures higher and lower than the spinodal temperature have been analyzed. By considering the time dependence of the VACF, the self-diffusion and shear viscosity coefficients, and the Maxwell relaxation time are determined, as well as their changes when crossing the spinodal. It is shown that the characteristic changes in the temperature dependences of the indicated kinetic coefficients allow the spinodal position to be determined with a high accuracy. A possibility to apply the proposed method to other low-molecular liquids is considered. As an example, nitrogen and oxygen are used, for which the averaged potential of intermolecular interaction has the Lennard-Jones form.

Ke ywords: self-diffusion coefficient, kinematic shear viscosity coefficient, Maxwell relaxation time, argon spinodal, averaged potential of molecular interaction.
\end{abstract}

\section{Introduction}

The velocity autocorrelation function (VACF) of molecules is a well-known research subject in molecular physics. Already as in works $[1,2]$, it was shown that the VACF behavior differs essentially from the exponential one typical of Markov processes. Of special interest was the discovery of universal long-time tails of VACF [1]. The physical nature of these tails was described in many works [3-14]. The special attention should be paid to work [12], where I.Z. Fisher introduced the concept of collective motion molecules in liquid and fulfilled the first estimation of the collective component $D_{c}$ of the self-diffusion coefficient $D_{s}$. Unfortunately, this approach obtained no further development because of the lack of reliable information in the literature at that time on the magnitude of the Maxwell relaxation time (MRT) $\tau_{\mathrm{M}}$ for viscous stresses.

A basis for overcoming this difficulty was formed in work [12]. In that work, an asymptotic long-time expression for the molecular VACF was obtained, which included the MRT as a parameter. However, at the

(C) N.P. MALOMUZH, K.S. SHAKUN, A.A. KUZNETSOVA, 2018

ISSN 2071-0194. Ukr. J. Phys. 2018. Vol. 63, No. 4 time when work [12] was published, the computational capabilities were very limited and insufficient to reproduce the behavior of the VACF of a molecule in liquid during the required time intervals and in a wide temperature range. Therefore, the results obtained in work [12] attracted no proper attention.

Below, we will demonstrate that, by applying the Lagrange theory of thermal hydrodynamic fluctuations $[12,15-17]$ to analyze the behavior of VACF at large times, the values of both the kinematic shear viscosity coefficient and the MRT can be obtained. Following this way, besides the determination of the ordinary self-diffusion coefficient, the determination of its collective component $D_{c}$ became possible.

The method developed in this work makes it possible to calculate all main kinetic coefficients for argonlike liquids $[18,19]$, in which the averaged potentials of molecular interaction have the Lennard-Jones form, by studying only the long-time asymptotics of the molecular VACF. The corresponding kinetic coefficients can be obtained by comparing the theoretical asymptotic expressions with the results of computer simulations. In such a way, the excessive calculations using the exact Kubo formulas [20] or some approximate methods can be avoided. 
In this work, the following kinetic coefficients are determined: 1) the self-diffusion coefficient and its collective component, 2) the kinematic shear viscosity $\nu$, and 3) the Maxwell relaxation time $\tau_{\mathrm{M}}$. The values of all indicated quantitieswere found by analyzing the long-time tails of the molecular VACF obtained for a number of isochores in a wide temperature range. As a result, it became possible to study the behavior of the indicated kinetic coefficients near the spinodal of argon. Proceeding from the obtained temperature dependences at isochores, a new method of determination of the spinodal position is proposed.

It should be emphasized that the results obtained are applicable both to argon and other low-molecular liquids with a nonspherical molecular symmetry. The extension of this approach to the case of lowmolecular liquids is possible owing to the permanent rotation of molecules, so that a lot of kinetic and thermodynamic parameters of the liquid can be determined with the help of averaged potentials. On the other hand, all the aforementioned kinetic coefficients are determined from the long-time VACF tails, whose shapes weakly depend on fine features of the interaction between molecules. Those features manifest themselves only within rather short time intervals. Since the averaged potentials for low-molecular liquids turn out similar to the Lennard-Jones potential and describe long-time processes in low-molecular liquids with a sufficient accuracy, liquid argon can serve as a reference system when determining the kinetic properties of more complicated liquids. Thus, all of the indicated kinetic coefficients of low-molecular liquids can be related to their counterparts for liquid argon by similarity relationships. This fact explains the particular attention that is paid to the computer-assisted simulation of long-time VACF tails for molecules of liquid argon.

\section{Some Features of Computer-Assisted Simulation}

In order to determine the VACF for molecules of liquid argon, krypton, and xenon, we simulated the dynamics of an ensemble of $N=50^{3}$ particles arranged in a cubic cell with imposed periodic boundary conditions. The simulation was carried out using the software package Gromacs 5.03 [21]. The interaction between the molecules was described with the help of the effective Lennard-Jones pair potential

$U(r)=4 \varepsilon\left[\left(\frac{\sigma}{r}\right)^{12}-\left(\frac{\sigma}{r}\right)^{6}\right]$

with the parameters taken from works $[22,23]$.

The applied model satisfied the following requirements:

- the linear size of the cell, $l_{1}$, should be much larger than the displacement radius $r_{c}$ of the interaction potential, $l_{1}>r_{c} \approx 7.5 \sigma$;

- the characteristic time of the sound propagation $\tau_{c}=l_{1} / c \sim 10^{-10} \mathrm{~s}$, where $c$ is the longitudinal sound velocity at the given density and temperature, should be much larger than the time interval of the formation of all fine features in the VACF (about $10^{-11} \mathrm{~s}$ );

- the pressure values calculated making use of the indicated $r_{c}$-value at the given temperature and density should be close to experimental ones [24].

The fulfillment of all mentioned requirements ensured the adequacy of the model to the analyzed molecular system. Using this model, the behavior of long-time VACF tails can be reproduced within time intervals that are required to determine the shear viscosity and the Maxwell relaxation time.

At the beginning of calculations, the molecules were arranged over the cell as a periodic structure with an interparticle distance corresponding to the considered density. The velocity field was generated according to the Maxwell distribution at the given temperature. At the initial simulation stage, the system relaxed to its equilibrium state during rather a long time, until the velocity and spatial distributions of molecules came to a mutual equilibrium. The resulting temperature of the system deviated from the initial value at that. In the course of the next stage, the temperature of the system was slowly (during 30-50 ps) returned back to a required value by means of the "soft temperature binding" (the NoséHoover thermostat $[25,26]$ with a large thermal inertia). Since this process was slow, the spatial and velocity distributions of molecules remained mutually consistent, so that there arose no significant fluctuations in the potential energy of the system. The last simulation stage was used to reproduce the time dependence of the VACF for liquid argon molecules. It was a simulation of the molecular dynamics in the NVE ensemble under equilibrium conditions and providing the required temperature and density.

ISSN 2071-0194. Ukr. J. Phys. 2018. Vol. 63, No. 4 
For the numerical solution of the dynamic equations, the Verlet algorithm with an integration step not exceeding 0.1 fs was applied. All the results presented below correspond to the averaging over a set of 20 different initial configurations.

In the context of the simulation problem and the interpretation of the data obtained, it should be noted that, in order to achieve the formulated tasks, the behavior of the VACF has to be studied on the times $t>10$ ps. However, the dynamic memory time [27] for the applied model is $\tau_{m} \approx 8 \mathrm{ps}$. The interval $\left(0, \tau_{m}\right)$ covers the region of the VACF fine structure, so that only the region of long-time tails turns out to be located beyond the indicated time interval.

Below, we will also demonstrate that there is a theoretical time limit for the applicability of the results of computer simulation to the description of the molecular VACF:

$t_{l} \sim \frac{2 \nu \tau_{\mathrm{M}}}{3 D_{s}} \sim 100 \mathrm{ps}$.

Here, $\nu$ is the kinematic shear viscosity coefficient, $\tau_{\mathrm{M}}$ the Maxwell relaxation time, and $D_{s}$ the self-diffusion coefficient. This threshold has the same order of magnitude as the threshold associated with the accumulation of calculation errors. The time $t_{l}$ can be shorter than the time accessible for a computer simulation.

\section{Determination of the Self-Diffusion Coefficient, Kinematic Shear Viscosity, and Maxwell Relaxation Time}

The self-diffusion coefficient $D_{s}$ was determined using the standard relations:

$D_{s}=\frac{1}{3} \int_{0}^{\infty} \phi_{\mathbf{V}}(t) d t$

where $\phi_{\mathbf{V}}(t)$ is the molecular VACF, and

$D_{s}=\lim _{t \rightarrow \infty} \frac{\Gamma(t)}{6 t}$,

where $\Gamma(t)$ is the mean square displacement of the molecule. The closeness of the values obtained on the basis of expressions (3) and (4) is one of the criteria that the computer model is proper [24].

In order to determine the kinematic shear viscosity and the Maxwel relaxation time, let us use the long-time asymptotic behavior of the VACF. Here, we will briefly summarize the results of works $[12,13,15-$ 17]. According to work [12], the asymptotic behavior of $\mathrm{VACF}$ is determined by the corresponding correlation function $\psi_{\mathbf{u}}(t)$ for the hydrodynamic velocity field $\mathbf{u}(\mathbf{r}, t)$ :

$\phi_{\mathbf{V}}(t) \rightarrow \psi_{\mathbf{u}}(t), \quad \psi_{\mathbf{u}}(t)=\langle\mathbf{u}(\mathbf{r}, \mathbf{t}) \mathbf{u}(\mathbf{r}, \mathbf{0})\rangle$.

It is well known that $\psi_{\mathbf{u}}(t)$ is connected with the correlation function for the spatial Fourier components of $\mathbf{u}(\mathbf{k}, t)$ by the formula

$\psi_{\mathbf{u}}(t)=\frac{1}{(2 \pi)^{3}} \int_{0}^{\infty}\left\langle\mathbf{u}^{*}(\mathbf{k}, \mathbf{t}) \mathbf{u}(\mathbf{k}, \mathbf{0})\right\rangle \mathbf{4} \pi \mathbf{k}^{2} \mathbf{d} \mathbf{k}$.

According to the results of works [15-17], the longtime tails of VACF are formed due to the transverse component of the hydrodynamic velocity field (below, the notation $\mathbf{u}$ will be used for it). In order to determine the Fourier components of the transverse field of hydrodynamic velocities, let us use the equation [28]

$\frac{\partial \mathbf{u}}{\partial t}+\tau_{\mathrm{M}} \frac{\partial^{2} \mathbf{u}}{\partial t^{2}}=-\nu k^{2} \mathbf{u}$

It is a consequence of the Maxwell model for the relaxing kinematic shear viscosity:

$\nu(\omega)=\frac{\nu(0)}{1+i \omega \tau_{\mathrm{M}}}$

A further modification of Eq. (7) is associated with the application of a more general approach to the problem (see works [29-34]). From Eq. (7), it follows that

$\mathbf{u}(\mathbf{k}, \mathbf{t})=\mathbf{u}(\mathbf{k}, \mathbf{0}) \times$

$\times \exp \left(-\frac{t}{2 \tau_{\mathrm{M}}}\left(1-\sqrt{1-4 \nu \tau_{\mathrm{M}} \mathbf{k}^{2}}\right)\right)$.

Substituting Eq. (8) into Eq. (6) and using the standard methods of statistical mechanics to calculate equilibrium correlation functions [28], we obtain

$\psi_{\mathbf{u}}(t)=\frac{k_{\mathrm{B}} T}{\pi^{2} \rho} \times$
$\times \int_{0}^{\infty} \exp \left(-\frac{t}{2 \tau_{\mathrm{M}}}\left(1-\sqrt{1-4 \nu \tau_{\mathrm{M}} \mathbf{k}^{2}}\right)\right) k^{2} d k$, 
Table 1. Experimental [40-42] and molecular dynamics values of $D_{s}$ and $\nu$ on the coexistence curve of argon

\begin{tabular}{|c|c|c|c|c|c|}
\hline$T, \mathrm{~K}$ & $\begin{array}{c}\rho, \\
\mathrm{g} / \mathrm{cm}^{3}\end{array}$ & $\begin{array}{c}D_{s}^{(\exp )} \times 10^{-5} \\
\mathrm{~cm}^{2} / \mathrm{s}\end{array}$ & $\begin{array}{c}D_{s}^{(\mathrm{MD})} \times 10^{-5} \\
\mathrm{~cm}^{2} / \mathrm{s}\end{array}$ & $\begin{array}{c}\nu^{(\exp )} \times 10^{-3} \\
\mathrm{~cm}^{2} / \mathrm{s}\end{array}$ & $\begin{array}{c}\nu^{(\mathrm{MD})} \times 10^{-3} \\
\mathrm{~cm}^{2} / \mathrm{s}\end{array}$ \\
\hline 0 & 1.379 & 2.36 & 2.304 & 1.796 & 1.673 \\
\hline 100 & 1.314 & 3.52 & 3.59 & 1.401 & 1.389 \\
\hline 110 & 1.243 & 4.85 & 4.79 & 1.134 & 1.191 \\
\hline 120 & 1.163 & 6.02 & 6.065 & 0.936 & 0.921 \\
\hline 130 & 1.068 & 7.43 & 7.48 & 0.78 & 0.744 \\
\hline 140 & 0.944 & 8.82 & 9.01 & 0.648 & 0.621 \\
\hline 150 & 0.68 & - & 12.45 & 0.519 & 0.597 \\
\hline
\end{tabular}

where $T$ is the temperature, $k_{\mathrm{B}}$ the Boltzmann constant, and $\rho$ the density. It can be shown (some details of calculations can be found in works $[15,35,36])$ that, at long times $\left(t \gg \tau_{\mathrm{M}}\right)$, the asymptotic behavior of $\psi_{\mathbf{u}}(t)$ looks like

$\psi_{\mathbf{u}}(t) \Rightarrow \frac{A}{t^{3 / 2}}\left[1-\frac{15}{4} \frac{\tau_{\mathrm{M}}}{t}+\ldots\right], \quad A=\frac{2 k_{\mathrm{B}} T}{(4 \pi \nu)^{3 / 2} \rho}$

From whence, it follows that the kinematic shear viscosity coefficient and the Maxwell relaxation time are determined by the expressions

$\nu=\frac{1}{4 \pi}\left(\frac{2 k_{\mathrm{B}} T}{B \rho}\right)^{2 / 3}, \quad B=\lim _{t \rightarrow \infty} \phi_{\mathbf{V}}(t) t^{3 / 2}$,

$\tau_{\mathrm{M}}=\frac{4}{15} \lim _{t \rightarrow \infty} t\left(1-\frac{\phi_{\mathbf{V}}(t) t^{3 / 2}}{B}\right)$.

Hence, making use of only the VACF $\phi_{\mathbf{V}}(t)$, whose profile is restored by a computer simulation $[21,24$, 37,38 , one can obtain two important parameters of the liquid. The obtained relations were derived using the hydrodynamic theory of fluctuations. Therefore, they have a universal character and can be applied to various low-molecular liquids.

\section{Results Obtained}

\section{by Computer Simulations}

In this section, the values calculatedfor the selfdiffusion coefficient of argon, its kinematic shear viscosity coefficient, and the Maxwell relaxation time, as well as the same results obtained for krypton and xenon using the similarity principle, are presented.

\subsection{Self-diffusion and kinematic shear viscosity coefficients}

The values for the self-diffusion and kinematic shear viscosity coefficients of liquid argon calculated by Eqs. (3) and (11) for the corresponding states on the coexistence curve are quoted in Table $1^{1}$. As follows from the table, the experimental and calculated values of $D_{s}$ and $\nu$ are in good agreement with each other. This circumstance testifies to the hydrodynamic origin of the long-time VACF tails. In other words, expression (10) is proper both from the qualitative and quantitative viewpoints.

\subsection{Isochoric kinematic shear viscosity of argon}

In this section, the values of the kinematic shear viscosity coefficient of argon calculated for several isochores are presented (see Fig. 1). As follows from the figure, the experimental and calculated values are close to each other, although this agreement is violated, when approaching the density corresponding to the triple point. For such densities, the VACF decreases more rapidly, and the relative determination error for $\nu(T)$ grows [24].

\subsection{Maxwell relaxation time for argon}

In this section, we consider the behavior of the Maxwell relaxation time for argon on the liquid-gas coexistence curve. The values obtained for $\tau_{\mathrm{M}}$ on the basis of Eq. ([10]) are presented in Table 2. As one can see, a quite satisfactory correspondence takes

${ }^{1}$ In this and other tables, the subscripts exp and MD denote the experimental data and the results obtained by a molecular dynamics simulation, respectively.

ISSN 2071-0194. Ukr. J. Phys. 2018. Vol. 63, No. 4 
Table 2. Comparison of the Maxwell relaxation times $\tau_{\mathrm{M}}^{(\mathrm{VACF})}$ for liquid argon on its liquid-gas coexistence curve obtained from the VACF with the results of other works

\begin{tabular}{|l|c|c|c|c|c|c|}
\hline$T, \mathrm{~K}$ & 90 & 100 & 110 & 120 & 130 & 140 \\
$\tau_{\mathrm{M}}^{(\mathrm{VACF})} \times 10^{-13}, \mathrm{~s}$ & $1.74 \pm 0.16$ & $1.67 \pm 0.14$ & $1.64 \pm 0.09$ & $1.62 \pm 0.11$ & $1.653 \pm 0.09$ & $1.771 \pm 0.08$ \\
$\tau_{\mathrm{M}} \times 10^{-13}, \mathrm{~s}[43]$ & 1.68 & - & 1.58 & 1.57 & 1.66 & 1.73 \\
$\tau_{\mathrm{M}} \times 10^{-13}, \mathrm{~s}[14]$ & $\approx 2.28$ & - & - & - & - & - \\
$\tau_{\mathrm{M}} \times 10^{-12}, \mathrm{~s}[44]$ & - & - & $\approx 2.1$ & $\approx 2.2$ & - & - \\
\hline
\end{tabular}

place between the MRT values obtained in work [43] and the results obtained from relation (12). In work [43], the MRT was determined by analyzing the autocorrelation function for transverse stresses. However, the ensemble of 4000 particles, which was used in the cited work, was not sufficiently complete to adequately describe the molecular interaction.

In early publications $[14,44]$, the standard definition of the Maxwell relaxation time, $\tau_{\mathrm{M}}=\eta / G_{\infty}$, was used as a rule; and the main attention was paid to the study of the high-frequency shear modulus $G_{\infty}$. In particular, in work [44], on the basis of a heuristic consideration, an equality was established between $G_{\infty}$ and the bulk elastic modulus $p_{\text {th }} /\left(T \gamma_{p}(T)\right)$ at high densities (here, $p_{\text {th }}$ is the so-called thermal pressure, i.e. the sum of the external and internal pressures in the system, and $\gamma_{p}(T)$ is the thermal expansion coefficient). Using the experimentally determined values of $\gamma_{p}(T)$, the authors of work [44] evaluated the Maxwell relaxation time.

Finally, let us discuss the temperature dependence of the MRT on the isochore $\rho=0.837 \mathrm{~g} / \mathrm{cm}^{3}$ (see Table 3 ). It is of interest that the MRT varies insignificantly on both the coexistence curve and the isochore. At the same time, the shear viscosity changes by almost an order of magnitude in the former case, but remains almost constant in the latter case.

\section{4. $D_{s-}-\nu$, and $\tau_{\mathrm{M}}$-values for argon-like liquids}

The values of $D_{s}$ and $\nu$ for krypton on the coexistence curve are presented in Table 4 . They were obtained from their counterparts for argon on the basis of the similarity principle:

$D_{i}\left(T_{i}\right)=\frac{\sigma_{i}}{\sigma_{\mathrm{Ar}}}\left(\frac{\varepsilon_{i}}{\varepsilon_{\mathrm{Ar}}} \frac{m_{\mathrm{Ar}}}{m_{i}}\right)^{1 / 2} D_{\mathrm{Ar}}\left(T_{\mathrm{Ar}}\right)$,

ISSN 2071-0194. Ukr. J. Phys. 2018. Vol. 63, No. 4
Table 3. Maxwell relaxation times

of liquid argon on the isochore $\rho=0.837 \mathrm{~g} / \mathrm{cm}^{3}$

\begin{tabular}{|l|c|c|c|}
\hline$T, \mathrm{~K}$ & 138 & 140 & 147 \\
$\tau_{\mathrm{M}} \times 10^{-13}, \mathrm{~s}$ & $2.26 \pm 0.09$ & $2.11 \pm 0.07$ & $2.14 \pm 0.11$ \\
\hline
\end{tabular}

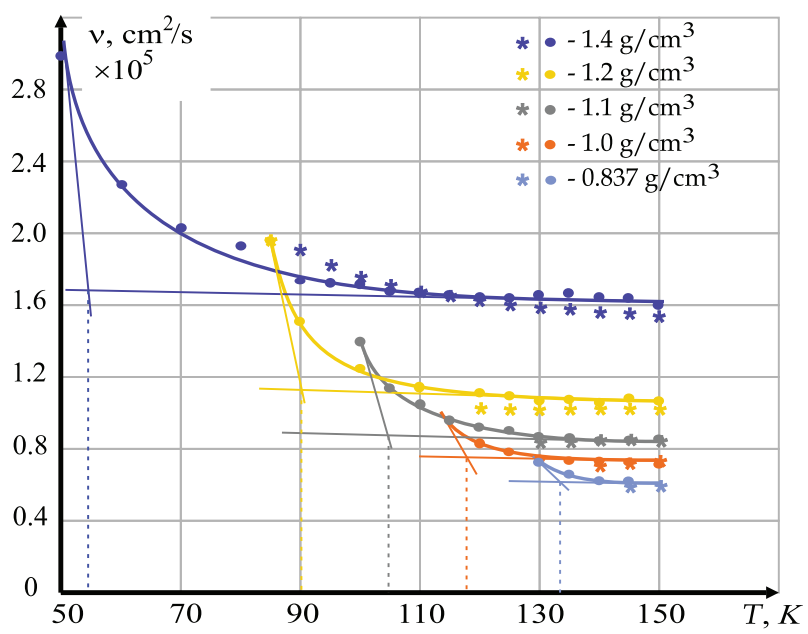

Fig. 1. Temperature dependences of the kinematic shear viscosity coefficient of argon along some isochores. Circles correspond to the results of a molecular dynamics simulation; stars correspond to experimental values [45]

$\nu_{i}\left(T_{i}\right)=\frac{\sigma_{i}}{\sigma_{\mathrm{Ar}}}\left(\frac{\varepsilon_{i}}{\varepsilon_{\mathrm{Ar}}} \frac{m_{\mathrm{Ar}}}{m_{i}}\right)^{1 / 2} \nu_{\mathrm{Ar}}\left(T_{\mathrm{Ar}}\right)$,

$\tau_{i}\left(T_{i}\right)=\frac{\sigma_{i}}{\sigma_{\mathrm{Ar}}}\left(\frac{\varepsilon_{\mathrm{Ar}}}{\varepsilon_{i}} \frac{m_{i}}{m_{\mathrm{Ar}}}\right)^{1 / 2} \tau_{\mathrm{Ar}}\left(T_{\mathrm{Ar}}\right)$,

$T_{i}=\frac{\varepsilon_{i}}{\varepsilon_{\mathrm{Ar}}} T_{\mathrm{Ar}}$

Here, $\varepsilon_{i}$ and $\sigma_{i}$ are the relevant parameters of the Lennard-Jones potential; $i=\mathrm{Kr}, \mathrm{Xe}, \ldots$; and $m_{i}$ is the molecular mass. 


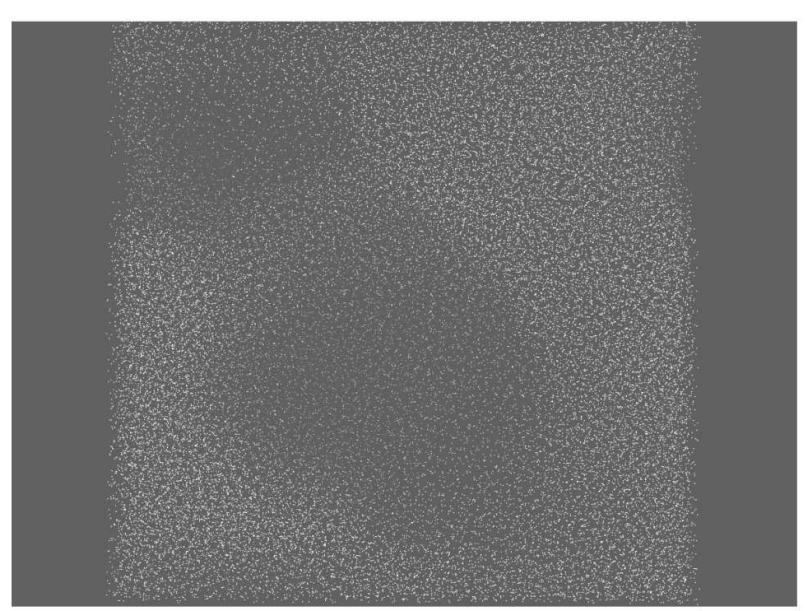

Fig. 2. Distribution of molecules over the volume of the elementary model cell. The density $\rho=0.837 \mathrm{~g} / \mathrm{cm}^{3}$, and the temperature $T=90 \mathrm{~K}$

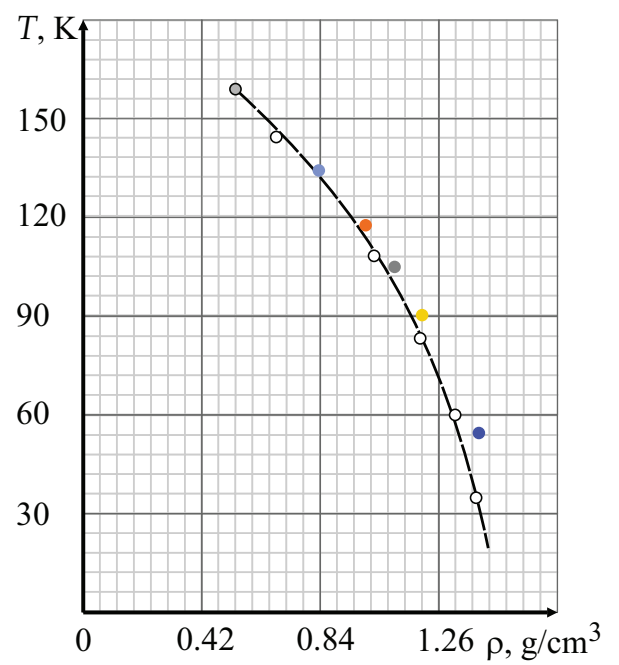

Fig. 3. Spinodal of argon. Hollow circles correspond to work [50], colored circles were obtained by analyzing the temperature dependences of the kinematic shear viscosity

The similarity relations for xenon can be calculated analogously.

\subsection{Spinodal of argon}

It should be noted that experimental data are available only for temperatures higher than the binodal of argon at the given density. The computer simulation makes it possible to calculate the parameters of metastable states located below the spinodal line in the phase diagram. In those states, the model system becomes stratified (see Fig. 2), and, owing to the in- fluence of high-density regions, the kinematic shear viscosity increases.

In addition, the self-diffusion coefficient reveals the opposite behavior, when crossing the spinodal. In this context, it should be noted that regions with different densities are not localized in the space in the model system. Therefore, the formalism of correlation functions is acceptable in this case [47]. Hence, the spinodal location can be determined by analyzing the temperature dependences of the kinetic coefficients in its vicinity.

In this work, we confine the calculation procedure to an approximate method by determining the location of the spinodal as the location of points corresponding to the intersection of high- and low-temperature asymptotics of the shear viscosity curves (see Fig. 1). Similar methods were used when studying the shear viscosity in liquids at their vitrification $[48,49]$. The arrangement of those points with respect to the spinodal curve was determined in work [50] and can be observed in Fig. 3.

It should be noted that the research of the temperature dependence of the self-diffusion coefficient gave rise to a similar result.

\section{6. $D_{s^{-}}, \nu-$, and $\tau_{M^{-}}$values \\ for diatomic nitrogen and oxygen on the coexistence curve}

Relations (13) for $D_{s}, \nu$, and $\tau_{\mathrm{M}}$ can be applied not only to monatomic liquids. In particular, it can be liquid nitrogen or oxygen as an example. In this case, the roles of $\varepsilon$ and $\sigma$ are played by the corresponding parameters of the averaged potential written in the

Table 4. Comparison of experimental $[45,46]$ and molecular dynamics values of $D_{s}, \nu$, and $\tau_{M}$ for krypton on its coexistence curve

\begin{tabular}{|l|c|c|c|c|}
\hline$T^{*}, \mathrm{~K}$ & 0.695 & 0.778 & 0.898 & 0.958 \\
$\rho_{\exp }, \mathrm{g} / \mathrm{cm}^{3}$ & 2.445 & 2.336 & 2.166 & 2.071 \\
$D_{s}^{(\exp )} \times 10^{-5}, \mathrm{~cm}^{2} / \mathrm{s}$ & 1.607 & 2.17 & 3.657 & 3.783 \\
$D_{s}^{(\mathrm{MD})} \times 10^{-5}, \mathrm{~cm}^{2} / \mathrm{s}$ & 1.431 & 1.951 & 3.511 & 4.112 \\
$\nu^{(\exp )} \times 10^{-3}, \mathrm{~cm}^{2} / \mathrm{s}$ & 1.837 & 1.369 & 0.986 & 0.861 \\
$\nu^{(\mathrm{MD})} \times 10^{-3}, \mathrm{~cm}^{2} / \mathrm{s}$ & 1.665 & 1.432 & 0.993 & 0.863 \\
$\tau_{\mathrm{M}} \times 10^{-13}, \mathrm{~s}$ & 2.09 & 2.00 & 1.92 & 1.87 \\
\hline
\end{tabular}

ISSN 2071-0194. Ukr. J. Phys. 2018. Vol. 63, No. 4 
Lennard-Jones form. According to work [51]

$$
\begin{aligned}
& \mathrm{N}_{2}: m=28, \quad \varepsilon / k_{\mathrm{B}}=95.05 K, \quad \sigma=3.698 \AA \\
& \mathrm{O}_{2}: m=32, \quad \varepsilon / k_{\mathrm{B}}=117.5 K, \quad \sigma=3.58 \AA \text {, }
\end{aligned}
$$

where $m$ is the mass of a molecule in atomic mass units. These values were obtained by reproducing the experimental values of the second virial coefficient with the help of the Lennard-Jones intermolecular potential. The $D_{s^{-}}, \nu$, and $\tau_{\mathrm{M} \text {-values calculated for }}$ liquid nitrogen according to Eqs. (13) and (14) are presented in Table 5.

The rather good agreement with the experimental data takes place for liquid oxygen as well. Analogously, by using the similarity principle, one can determine the position of the spinodal for liquid nitrogen and other liquids consisting of dumbbell-like molecules.

The deviations of the kinematic shear viscosity values from the corresponding values for argon at various temperatures are depicted in Fig. 4. One can see that slight deviations are observed even for monatomic liquids. This circumstance is probably connected with different role of many particle effects, in particular, with small dimerization near the critical point [51]. In the case of dumbbell-like molecules, the deviation is some larger owing to the angle correlation effect. This effect will be considered elsewhere.

\section{Discussion of the Results Obtained}

In this work, we showed that much useful information can be obtained by analyzing the VACF of molecules. The possibilities of the proposed method are illustrated by the example of liquid argon. The asymptotic expression (10), which is suitable to restore the asymptotic behavior of the VACF at large times on the basis of the results obtained by the molecular dynamics methods, is used to calculate the kinematic shear viscosity coefficient and the Maxwell relaxation time. The results obtained are in good agreement with the data of known experimental works (see Tables 1 to 3 ). The temperature dependences of the kinetic coefficients and the MRT for other liquefied inert gases (see Table 4) are determined by applying the similarity method. Generally speaking, this is an expected result.

A satisfactory determination of the kinetic coefficients and the MRT was also carried out for twoatomic liquids of the $\mathrm{N}_{2}$ type (Table 5). This re-

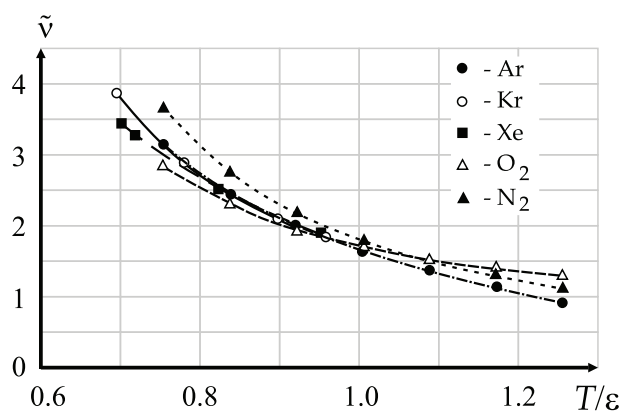

Fig. 4. Experimental temperature dependences of the kinematic shear viscosity coefficients of argon, krypton, xenon, oxygen, and nitrogen $[45,46]$ on the corresponding coexistence curve. $\tilde{\nu}=\nu /\left(\sigma \varepsilon^{1 / 2} m^{-1 / 2}\right)$

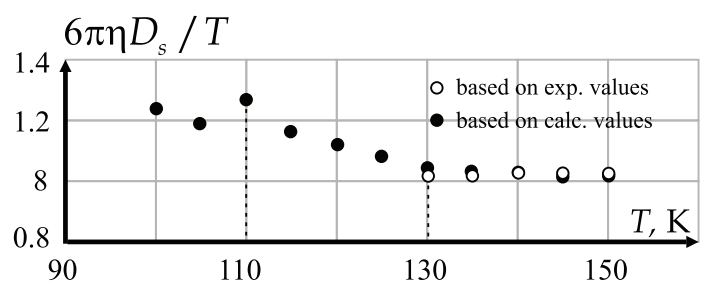

Fig. 5. Temperature dependence of the quantity $C=6 \pi \times$ $\times \eta D_{s} / T$ for liquid argon with $\rho=1 \mathrm{~g} / \mathrm{cm}^{3} . \eta$ is the dynamic viscosity coefficient at the considered density

Table 5. Experimental [46] and molecular dynamics values of $D_{s}, \nu$, and $\tau_{\mathrm{M}}$ for liquid nitrogen on its coexistence curve

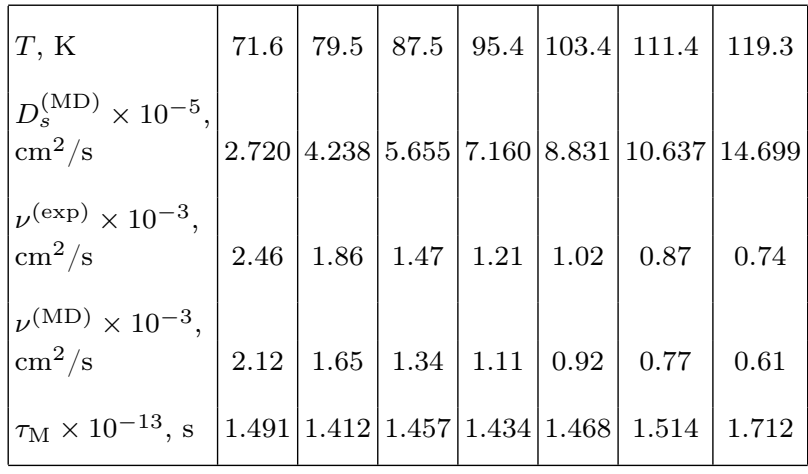

sult is less trivial. It means that the corresponding kinetic coefficients can be determined with a sufficient accuracy using the averaged potentials. In fact, the averaging of the intermolecular potential becomes possible due to the rotational motion of the molecules. 
Hence, the proposed method makes it possible to determine all main kinetic coefficients for liquids, in which the averaged potential of interaction between the molecules has the Lennard-Jones form. It is important that the final result can be obtained by studying only the long-time asymptotic behavior of the molecular velocity autocorrelation function. The latter is one of the simplest and well-studied subjects in molecular dynamics. The corresponding kinetic coefficients are determined by comparing the results of the theoretical analysis of the VACF asymptotics with the results of computer simulations. In such a way, excessive calculations by the exact Kubo formulas can be avoided.

The applicability of the averaged intermolecular potential to the considered two-atomic liquids can be estimated from the dependences shown in Fig. 4. The closer the dependences $\tilde{\nu}(T)$ for the examined liquid and argon are located to each other, the more accurately the averaged potential describes the interaction between the molecules. The anisotropic part of the intermolecular potential is a result of the dipole-dipole, dipole-quadrupole, and quadrupole-quadrupole interactions. The former is significant near the triple point and in supercooled states. The latter is responsible for orientational correlations and clustering effects. Finally, the dipole-quadrupole interaction is important in a vicinity of the critical point. The specific features of the kinetic coefficients for twoatomic liquids are determined by the quadrupole interaction. Therefore, the difference from the kinetic coefficients of argon is insignificant: it does not exceed $15 \%$. However, the influence of the dipole-dipole interaction is considerable. For instance, this interaction in water is much stronger and will be considered separately.

It should be noted that, for the parameters $\nu$ and $\tau_{\mathrm{M}}$ to be determined with a satisfactory accuracy, the VACF evolution was calculated to times of about 20 ps. In this connection, important is the issue concerning the applicability area of hydrodynamic correlations to describe the VACF tails. An exhaustive answer to this question has to be found in the framework of the Lagrange theory of thermal hydrodynamic fluctuations $[12,13,15-17]$. It is evident that the hydrodynamic description of the long-time VACF asymptotics is possible for times, for which the application of the concept of a Lagrange particle involving the given molecule into the hydrodynamic motion is proper. By the order of magnitude, the lifetime of a Lagrange particle can be determined from the relation

$t_{l} \sim \frac{r_{\mathrm{L}}^{2}}{6 D_{s}}$

where $r_{\mathrm{L}}$ is the corresponding radius of a Lagrange particle. Assuming, in accordance with the results of work [17], that $r_{\mathrm{L}}=2 \sqrt{\nu \tau_{\mathrm{M}}}$, we obtain

$t_{l} \sim \frac{2 \nu \tau_{\mathrm{M}}}{3 D_{s}}$

The numerical value of $t_{l}$ never exceeds $10^{-10} \mathrm{~s}$. This fact means that the application of hydrodynamic methods, while describing the long-time VACF tails up to $20 \mathrm{ps}$, is physically proper.

The temperature dependence of the quantity $C=$ $=6 \pi \eta D_{s} / T$, which was studied in work [52] in the case of Lennard-Jones liquids, is depicted in Fig. 5 for argon with the density $\rho=1 \mathrm{~g} / \mathrm{cm}^{3}$. It is noteworthy that the temperature $T_{B}=130 \mathrm{~K}$, which divides the temperature interval into the left- and righthand (where $C$ is almost constant) sections, coincides with the position of the binodal at the considered density. The temperature $T_{S}=110 \mathrm{~K}$, at which a violation of the $C(T)$-monotonicity is observed, corresponds to the spinodal. This circumstance deserves to be studied in more details.

The value and the temperature dependence of the collective component in the self-diffusion coefficient near the spinodal should also be discussed separately. The main attention will be focused to the properties of water.

To summarize, we would like to express our sincere gratitude to Prof. G.E. Norman for a detailed discussion of the simulation methods and the results obtained. This and many other related works could not be carried out without friendly and competent interest of Academician Leonid Bulavin. We express our heartful gratitude to him and to our co-author Vitalii Bardik.

1. B.J. Alder, T.E. Wainwright. Velocity autocorrelations for hard spheres. Phys. Rev. Lett. 18, 988 (1967).

2. B.J. Alder, T.E. Wainwright. Decay of the velocity autocorrelation functions. Phys. Rev. A 1, 18, (1970).

3. M.H. Ernst, E.H. Hauge, J. van Leewen. Asymptotic time behavior of correlation functions. Phys. Rev. Lett. 25, 1254 (1970).

ISSN 2071-0194. Ukr. J. Phys. 2018. Vol. 63, No. 4 
4. M.H. Ernst, E.H. Hauge, J. van Leewen. Hydrodynamic theory of the velocity correlation function. Phys. Lett. A 34, 419 (1971).

5. M.H. Ernst, E.H. Hauge, J. van Leewen. Asymptotic time behavior of correlation functions. I. Kinetic terms. Phys. Rev. A 4, 2055 (1971).

6. K. Kawasaki. Long time behavior of the velocity autocorrelation function. Phys. Lett. A 32, 379 (1970).

7. T. Gaskell, N.H. March. Non-analyticity of frequency spectra in classical liquids. Phys. Lett. A 33, 460 (1970).

8. M.H. Ernst, J.R. Dorfman. Nonanalytic dispersion relations in classical fluids: I. The hard-sphere gas. Physica 61, 157 (1972).

9. J.R. Dorfman, E.G.D. Cohen. Time correlation functions. Phys. Rev. A 12, 292 (1975).

10. N.N. Bogolyubov. On stochastic processes in dynamic systems. Elem. Chast. At. Yadr. 9, 501 (1978) (in Russian).

11. P. Resibois, M. De Leener. Classical Kinetic Theory of Fluids (Wiley, 1978)

12. I.Z. Fisher. Hydrodynamic asymptotic characteristics of the autocorrelation function for the velocity of a molecule in a classical liquid. Sov. Phys. JETP 61, 1647 (1971)

13. I.Z. Fisher, A.V. Zatovsky, N.P. Malomuzh. Asymptotics of the angular velocities autocorrelation function of a molecule for the liquid argon. Sov. Phys. JETP 65, 297 (1973).

14. A.R. Dexter, A.J. Matheson. Elastic moduli and stress relaxation times in liquid argon. J. Chem. Phys. 54, 203 (1971).

15. T.V. Lokotosh, N.P. Malomuzh. Lagrange theory of thermal hydrodynamic fluctuations and collective diffusion in liquids. Physica A 286, 474 (2000).

16. T.V. Lokotosh, N.P. Malomuzh. Manifestation of the collective effects in the rotational motion of molecules in liquids. J. Mol. Liq. 93, 95 (2001).

17. T.V. Lokotosh, N.P. Malomuzh, K.S. Shakun. Nature of oscillations for the autocorrelation functions for translational and angular velocities of a molecule J. Mol. Liq. 96-97, 245 (2002).

18. L.A. Bulavin, D.A. Gavryushenko, and V.M. Sysoev, Molecular Physics (Znannya, 2006) (in Ukrainian).

19. L.A. Bulavin, Neutron Diagnostics of Liquid Matter State (Institute for Safety Problems of Nuclear Power Plants, 2012) (in Ukrainian).

20. D. Zubarev, V. Morozov, G. Ropke. Statistical Mechanics of Nonequilibrium Processes (Wiley, 1997).

21. D. van der Spoel, E. Lindahl, B. Hess, G. Groenhof, A.E. Mark, H.J.C. Berendsen. Gromacs: fast, flexible and free. J. Comp. Chem. 26, 1701 (2005).

22. W.F. van Gunsteren, S.R. Billeter, A.A. Eising, P.H. Hunenberger, P. Kruger, A.E. Mark, W.R.P. Scott, I.G. Tironi. Biomolecular Simulation: The GROMOS96 Manual and User Guide (Hochschulverlag AG an der ETH, 1996).

23. C. Oostenbrink, A. Villa, A.E. Mark, W.F. van Gunsteren. A biomolecular force field based on the free enthalpy of hy- dration and solvation: The GROMOS forcefield parameter sets 53A5 and 53A6. J. Comput. Chem. 25, 1656 (2004).

24. D. Frenkel, B. Smit. Understanding Molecular Simulation: from Algorithms to Applications (Academic Press, 2001).

25. S. Nosé. A molecular dynamics method for simulations in the canonical ensemble. Mol. Phys. 52, 255 (1984).

26. W.G. Hoover. Canonical dynamics: Equilibrium phasespace distributions. Phys. Rev. A 31, 1695 (1985).

27. A.Yu. Kuksin, I.V. Morozov, G.E. Norman, V.V. Stegailov, I.A. Valuev. Standards for molecular dynamics modelling and simulation of relaxation. Mol. Simulat. 31, 1005 (2005).

28. P.M. Morse, H. Feshbach. Methods of Theoretical Physics (McGraw-Hill, 1953), Vol. 1.

29. O.A. Grechanyi. Stochastic Theory of Irreversible Processes (Naukova Dumka, 1989) (in Russian).

30. Yu.V. Slyusarenko. Influence of fluctuations on hydrodynamic asymptotics of Green's functions. Ukr. Fiz. Zh. 28, 774 (1983) (in Russian).

31. A.I. Sokolovsky. Projection formulation of the Bogolyubov reduced description method and its application to fluctuation kinetics. Ukr. J. Phys. 45, 545 (2000).

32. A.I. Sokolovsky. Reduced description of nonequilibrium processes and correlation functions. Divergences and nonanalyticity. Condens. Matter Phys. 9, 415 (2006).

33. S.V. Peletminsky, Yu.V. Slusarenko. On the theory of long wave nonequilibrium fluctuations. Physica A 210, 412 (1994).

34. S.V. Peletminskii, Yu.V. Slyusarenko, A.I. Sokolovsky. Kinetics and hydrodynamics of long-wave fluctuations under external random force. Physica A 326, 412 (2003).

35. L.A. Bulavin, T.V. Lokotosh, N.P. Malomuzh. Role of the collective self-diffusion in water and other liquids. J. Mol. Liq. 137, 1 (2008).

36. T.V. Lokotosh, M.P. Malomuzh, K.M. Pankratov, K.S. Shakun. New results in the theory of collective selfdiffusion in liquids. Ukr. Fiz. Zh. 60, 697 (2015) (in Ukrainian).

37. M.E. Soloviev, M.M. Soloviev. Computer Chemistry (Solon-Press, 2005) (in Russian).

38. L.A. Bulavin, N.V. Vygornitskii, N.I. Lebovka. Computer Modeling of Physical Systems (Intellekt, 2011) (in Russian).

39. L.D. Landau, E.M. Lifshitz. Statistical Physics (Pergamon Press, 1980).

40. J. Naghizadeh, S.A. Rice. Kinetic theory of dense fluids. $\mathrm{X}$. Measurement and interpretation of self-diffusion in liquid $\mathrm{Ar}, \mathrm{Kr}, \mathrm{Xe}$, and $\mathrm{CH}_{4}$. J. Chem. Phys. 36, 2710 (1962).

41. R. Laghaei, A.E. Nasrabad, Byung Chan Eu. Generic van der Waals equation of state, modified free volume theory of diffusion, and viscosity of simple liquids. J. Phys. Chem. B 109, 5873 (2005).

42. B.A. Younglove, H.J.M. Hanley. The viscosity and thermal conductivity coefficients of gaseous and liquid argon. J. Phys. Chem. Ref. Data 15, 1323 (1986). 
43. R. Hartkamp, P.J. Daivis, B.D. Todd. Density dependence of the stress relaxation function of a simple fluid. Phys. Rev. E 87, 032155 (2013).

44. P.S. van der Gulik. The linear pressure dependence of the viscosity at high densities Physica A 256, 39 (1998).

45. NIST Standard Reference Database 69: NIST Chemistry WebBook; http://webbook.nist.gov/chemistry/fluid/

46. B.P. Nikolskiy. Chemical Handbook (Chemistry, 1965), Vol. 1.

47. N.P. Malomuzh, K.S. Shakun. Specific properties of argonlike liquids near their spinodals. J. Mol. Liq. 235, 155 (2017).

48. W.T. Laughlin, D.R. Uhlmann. Viscous flow in simple organic liquids. J. Phys. Chem. 76, 2317 (1972).

49. A.R. Ubbelohde. Melting and Crystal Structure (Oxford Univ. Press, 1965).

50. A.Yu. Kuksin, G.E. Norman, V.V. Stegailov. The phase diagram and spinodal decomposition of metastable states of Lennard-Jones system. High Temp. 45, 37 (2007).

51. P.V. Makhlaychuk, V.N. Makhlaychuk, N.P. Malomuzh. Nature of the kinematic shear viscosity of low-molecular liquids with averaged potentials of Lennard-Jones type. J. Mol. Liq. 225, 577 (2016).
52. N. Ohtori, Y. Ishii. Explicit expression for the StokesEinstein relation for pure Lennard-Jones liquids. Phys. Rev. E 91, 012111 (2015).

Received 27.12.17. Translated from Ukrainian by O.I. Voitenko

М.П. Маломуж, К.С. Шакун, Г.О. Кузнєцова

НОВІ МОЖЛИВОСТІ АНАЛІЗУ

АВТОКОРЕЛЯЦЙНОЇ ФУНКЦЇ ШВИДКОСТІ МОЛЕКУЛ РІДИНИ

Р е $з$ ю м е

Робота присвячена аналізу довготривалих хвостів автокореляційної функції швидкості (АКФШ) молекул рідкого аргону при температурах, вищих та нижчих за спінодаль. Аналізуючи часову залежність АКФШ визначено значення коефіцієнтів самодифузії, зсувної в'язкості, максвелівського часу релаксації, а також їх зміну при перетинанні спінодалі. Показано, що характерні зміни температурних залежностей згаданних кінетичних коефіцієнтів дозволяють визначити положення спінодалі з високою точністю. Розглянута можливість поширення запропанованого методу на інші низькомолекулярні рідини на прикладі азоту та кисню, для яких усереднений потенціал міжмолекулярної взаємодії має ленард-джонсівську форму. 